\title{
Preliminary results of a randomised controlled trial to evaluate the efficacy of a dietary and physical activity intervention in prostate cancer patients receiving androgen deprivation therapy
}

\author{
F. Haseen ${ }^{1,2}$, R. F. O’Neill ${ }^{2}$, L. J. Murray ${ }^{2}$, J. M. O’Sullivan ${ }^{3}$ and M. M. Cantwell ${ }^{2}$ \\ ${ }^{1}$ Population Health Section, Institute of Applied Health Sciences, University of Aberdeen, Aberdeen AB25 2ZD, UK, ${ }^{2}$ Centre \\ for Public Health, Queen's University Belfast, Belfast BT 12 6BJ, UK and ${ }^{3}$ Centre for Cancer Research and Cell Biology, \\ Queen's University Belfast, Belfast BT9 7BL, UK
}

\begin{abstract}
Androgen deprivation therapy (ADT) is a common treatment for prostate cancer that may be associated with unfavourable changes in body composition, including increased fat and decreased lean mass; and increased fatigue. These changes can negatively affect quality of life. The impact of a diet and physical activity intervention on these side effects related to ADT has not been examined in any study to date. Therefore, this study investigated whether a 6-month diet and physical activity intervention is effective at reducing ADT related side effects and improve overall quality of life in prostate cancer patients.

Prostate cancer patients prescribed ADT treatment for at least 6 months were recruited from Belfast Cancer Centre, Northern Ireland and randomised either to receive the dietary modification and physical activity intervention or standard care (control group) with a $1: 1$ allocation ratio. The intervention consisted of an individually tailored home-based one-to-one counselling delivered by a nutritionist to help the patients in the intervention group to modify their diet to meet healthy eating guidelines and achieve 30 min of brisk walking, five or more days per week. The primary outcomes were body composition, fatigue and quality of life at baseline, 3 and 6 months. Changes in dietary intake, perceived stress, functional capacity and energy expenditure were investigated as secondary outcomes.

This preliminary analysis included forty-two patients (twenty-one patients in each group) who have completed a 6-month follow-up. Analysis of covariance was used to compare outcomes for groups at 3 and 6 months adjusted for baseline values. Statistically significant differences were noted between the intervention and the control group for body composition. The patients in the intervention group had a statistically significant reduction in mean body weight $(-3.8 \pm 3.7 \mathrm{~kg})$, while weight increased in the control group $(0.8 \pm 2.8 \mathrm{~kg})$ $(P<0.001)$. Both BMI $(P<0.001)$ and waist-hip ratio $(P=0.01)$ decreased significantly in the intervention group compared with the control group. The mean changes in $\%$ fat and total fat were $-2.2 \pm 1.3 \%$ and $-3.3 \pm 2.6 \mathrm{~kg}$, respectively, in the intervention group compared with $1.1 \pm 1.6 \%$ and $1.1 \pm 2.1 \mathrm{~kg}$ in the control group $(P<0.001)$. There was a reduction in mean fatigue scores in the intervention group $(-1.8 \pm 8.5)$ compared with the controls $(2.3 \pm 9.1)$, with no significant difference between the groups. Results on quality of life scores showed no statistically significant differences between the groups on any general quality of life subscales; however, the prostate cancer specific subscale showed a worsening of prostate cancer related symptoms in the control group compared with the intervention group $(P=0.001)$. Patients undergoing intervention showed an increase energy expenditure $(P=0.04)$ and functional capacity $(P=0.002)$ compared with the control group. Intervention patients had significantly improved their diet by decreasing energy, fat, saturated fat, cholesterol sugar and salt consumption compared with the controls. Furthermore, the mean intake of fruits and vegetables increased in the intervention group $(1.4 \pm 1.5$ servings/d), while there was a decrease in the control group $(-0.07 \pm 1.5$ servings/d) $(P=0.002)$.

Preliminary results suggest that a 6-month dietary and physical activity intervention may have favourable effects on ADT related side effect, especially in reducing the negative body composition changes among prostate cancer patients. However, only $44.7 \%$ (total sampleninety-four patients) of patients completed the intervention to date and the analysis was underpowered to detect significant differences in fatigue and quality of life. The results of the completed study are eagerly awaited and will help to inform future guidelines for prostate cancer patients treated with ADT.
\end{abstract}

